Canadian Journal of Family and Youth, 8(1), 2016, pp 1-17

ISSN 1718-9748 @ University of Alberta

http://ejournals.library.ualberta.ca/index/php/cjfy

\title{
Peer Support and Youth Recovery: A Brief Review of the Theoretical Underpinnings and Evidence
}

\author{
Jessica Barton and Joanna Henderson
}

\begin{abstract}
This article provides a brief review of the theoretical underpinnings and initial evidence regarding peer support, with a specific interest in peer support for youth with addiction and mental health illnesses. Peer support can be thought of in terms of supporting prevention of difficulties, often emphasizing naturalistic opportunities, or as a specific component of an intervention targeting individuals with identified difficulties. In addition, there are peer-led and peer-implemented treatment approaches that have been evaluated. This form of peer support involves an asymmetrical relationship whereby a trained worker, who has gone through the process of recovery, assists other individuals who are not as far along in their recovery. Interaction with peers who have lived experience is thought to offer hope and enhance motivation for individuals considering or attempting change. These different forms of peer support have all been shown to hold promise for improving clinical outcomes but the literature in general has been hampered by limitations in study methods and design. Future studies will need to address these historical challenges for the potential of peer support to enhance outcomes to be fully realized.
\end{abstract}

Jessica Barton is a Canadian born psychology student at University College London in England. She completed a 7-week Research Program placement at the Centre for Addiction and Mental Health in the summer of 2014. She is currently working on her undergraduate thesis investigating the effects of a web-based intervention involving selfaffirmation and risk-appraisal on alcohol consumption for at-risk youth. Joanna Henderson is a Clinician Scientist at the Centre for Addiction and Mental Health and Assistant Professor of Psychiatry at the University of Toronto. Dr. Henderson's research interests include youth substance use and mental health, trauma, youth risk-taking, helpseeking behaviour, and innovative models of knowledge translation and exchange. Selected publications_include: 1) Henderson J, Brownlie EB, Rosenkranz S, Chaim G, \& Beitchman J. "Addressing the research-practice gap through stakeholder involvement in grant proposal development." Journal of the Canadian Academy of Child and Adolescent Psychiatry. 22(4), 268-274; 2) Rosenkranz S, Muller RT, \& Henderson J. "The role of complex PTSD in mediating childhood maltreatment and substance abuse severity among youth seeking substance abuse treatment." Psychological Trauma: Theory, Research, Practice \& Policy, 2013. doi: 10.1037/a0031920; and 3) Henderson J, Milligan K, Niccols A, Thabane L, Sword W, Smith A, \& Rosenkranz S. "Reporting of feasibility factors in publications on integrated treatment programs for women with substance abuse issues and their children: A systematic review and analysis." Health Research Policy and Systems. 2012:10(37).

Acknowledgement: The authors are grateful to Melissa Griffin for her helpful comments on this manuscript. 


\section{Introduction}

Peer support is generally defined as a structured relationship where a trained worker or volunteer, who has gone through a process of recovery, assists other people with mental health problems to identify and achieve life goals as part of their own experience with the recovery process (Davidson et al., 1999). People coping with severe mental illnesses could benefit from having peer role models who have been successful in managing their own disorders (Deegan, 1993). Furthermore, interactions with recovered individuals may offer hope and motivation for a brighter future (Davidson et al., 1999). Additionally, it is thought that peer support counselors offer visible examples of individuals who are countering stigmas about mental illness by regaining a sense of purpose and agency in their life, and by successfully reintegrating themselves back into their communities. Accordingly, peer support can be seen to be providing enhanced guidance for recovery from people who are perceived to have a broad range of firsthand knowledge and, as a result, credibility in the field (Carling, 1995). Notably, those involved in the mental health consumer movement consider peer support to also be an especially effective resource for those in recovery from substance abuse disorders, and highlights that substance abuse treatment communities have long believed in the efficacy of the firsthand knowledge of recovered individuals (Davidson, Chinman, Sells \& Rowe, 2006; Jacobson \& Curtis, 2000).

This paper seeks to describe selected theoretical perspectives that underlie peer support interventions and examine supporting evidence with regards to recovery in addiction and mental health. Despite being a strongly advocated practice, there are few well-described peer support models that are widely used in treatment programs (Davidson, Chinman, Sells, \& Rowe, 2006).

\section{Psychosocial Theory}


Peer Support and Youth Recovery

The psychological theories that underpin peer support provide a robust theoretical basis to understanding the potential of peer support to be effective. In particular, Festinger's Social Comparison Theory and Bandura's Social Learning Theory offer theoretical rationales for the value of peer support. Social Comparison Theory proposes that individuals self-evaluate based on the comparison of their own beliefs and desires against those of another person's. In this way, individuals define and reduce uncertainties about themselves. Individuals seek 'selfenhancement' to improve their self-esteem, which involves either an upward or downward social comparison (Wood, 1989).

Downward comparisons are made when a person looks down on another social group that they believe to be worse off to elevate self-regard (Wills, 1981) while in contrast, upward comparison usually serves to do the opposite and lower self-regard. Collins (1996), however, has found that this is not always the case. Upward social comparisons, to a comparison group that is perceived to be superior to one's own, may occur in order to create a more positive perception of one's own reality. Research has shown that upward social comparisons may increase one's motivation to improve oneself (Taylor \& Lobel, 1989). By placing at-risk individuals with peers who have successfully changed their habits, they too, may aspire toward a positive behavior change (Glass, DeLeon, Bassuk \& Berkman, 2006). Moreover, Festinger argued that the tendency to compare oneself to another decreases as the person's opinions and abilities are perceived to be more divergent. In the context of counseling this may mean that individuals are less likely to view counselors who differ in substantial ways from themselves as role models. Peer support workers, on the other hand, may be perceived by individuals to be more similar as a result of their shared lived experiences and may provide common ground between the two individuals upon which to change. 
Another model that supports the potential efficacy of peer support is Bandura's Social Learning Theory. This behaviorist theory posits that people learn through observing another's behaviour and attitudes, as well as the outcomes of that behaviour. In this way, behavior is modeled and can serve as a template, or guide, for future action. Social Learning Theory draws heavily on the concept of the live model, in which an observed person demonstrates the desired behavior and produces visible consequences for their actions. In this way, peer support may act as a vicarious learning experience that motivates an individual to replicate such behavior (Miller, 2010). Individuals who value the perceived effects of changed lifestyles have an incentive to change if they believe (a) that their current lifestyle poses threats to a personally valued outcome, such as their own health, (b) that particular behavioral changes will reduce the threats, and (c) that they are personally capable of adopting the new behaviors. Peer support workers may offer explicit and indirect evidence in these areas, thereby promoting change.

\section{Supportive communities: An example of both theories in practice.}

Supportive community organizations such as Permanent Supportive Housing (PSH) and Alcoholics Anonymous (AA) lend support for both Social Comparison and Social Learning Theory. PSH provides individuals with alcohol and drug addictions a safe space in which to receive case management, job skill training, community support services and alcohol and drug management group therapy programs. Both trained social workers and members of the PSH community work together to build a healthier lifestyle. These peer support-based communities provide individuals with an opportunity to give and receive support, and reflect on explicit beliefs that are considered essential to recovery. Within these communities, psychosocial theories postulate that positive social comparisons can be made, as well as positive modeling by recovered individuals. The guidelines and collective effort to remain 'healthy' established by 
peer support communities guide how individuals ultimately relate to themselves, peers, significant others, and to the larger community (Boisvert, Martin, Grosek \& Claire, 2008).

\section{Peer Support in Primary Prevention and Education}

Today, most adolescents are exposed to drug or alcohol use, and few are invulnerable to their influence (Boak, Hamilton, Adlaf \& Mann, 2013). Substance use behaviors may be influenced by perceived closeness with the youth's family or peers, as well by being exposed to others who are using substances (Shillington et al., 2005). There is a growing body of evidence that suggests that support from family and friends can act as a preventative measure for youth at risk of developing substance disorders (Birckmayer, Holder, Yacoubian \& Friend, 2004).

Moreover, during adolescence the protective effects of peer support seem to grow in importance as youth become less strongly influenced by their parents (O’Connel, Boat \& Warner, 2009). Work by Vaughan, Foshee and Ennet (2010) demonstrates the capacity of peer support to be protective regarding adolescent substance abuse. In Vaughan et al.'s study, 3,444 16 year olds participated at 6-month intervals over the course of two years. To measure 'peer support', participants listed the names of five friends that they were close to over the course of the previous 6-month wave, and reported how close they felt in each relationship $(0=$ not close at all to $3=$ very close). Participants also reported the number of extracurricular activities that they engaged in with friends over the past week. Results showed that more frequent interaction with peers who were deemed trustworthy and helpful were related to fewer depressive symptoms and incidents of drug use. This research supports the relevance of social support to mental health and substance abuse during adolescence. Since the study is focused primarily on the importance of naturally occurring peer support, it does not, however, suggest an intervention method to help adolescents who engage less frequently in social situations. Furthermore, negative aspects of 
peer relations, such as conflict, were not measured and may be especially relevant to drug abuse evaluations.

Research conducted by Eggert and colleagues (1994) addresses these issues, by providing empirical evidence for interventions that promote social network development. Youth were either randomly assigned to a regular school schedule (control condition), or to a schedule that included a Personal Growth Program (PGP) as part of one of the six regular classes. The PGP was developed to emphasize group-support training and life-skills interventions, in the hopes of intensifying positive peer-group relations. Academic performance, class absences, self-esteem, deviant peer bonding and various aspects of drug use (e.g., change, control and consequences) were measured. The PGP was associated with significantly reduced drug related problems and consequences at the end of the program at five months and at a ten month follow up.

Participation in the PGP was also related to improved mean GPA scores and self-esteem as well as a reduction in the number of deviant peer-bonding relationships. This intervention provides evidence for the importance of peer and community relationships in deterring poor health behaviors, and for the potential of education-based initiatives to foster better peer relations.

As mentioned previously, Festinger (1954) postulates that the more in-sync one's beliefs and attitudes are with those of another person, the more likely one is to make a social comparison, which may ultimately confer behavior change. Ward, Hunter and Power (1997) found that peer education and interventions can be very successful in disseminating health information to hidden or hard-to-reach populations, by passing on harm-reduction knowledge that promotes healthier lifestyles. Individuals were recruited from different community settings to undertake peer education activities within the context of their social lives, targeting youth between the ages of 15 and 25. The program was effective in promoting and changing health 
behaviours, and suggests that peer education is a practical model of service delivery. Research demonstrates that young people rarely approach formal drug services for assistance, and that peer education may be a more viable method of intervention (Ward et al., 1997).

\section{Peer Support as an Intervention Component}

In the studies reviewed previously, the focus was on efforts outside the treatment system and with individuals who have no formal diagnosis of mental health or substance abuse problems. The impact of peer support, however, may have different effects on those with and without identified mental health and/or substance abuse problems. Accordingly, the following section will discuss the role of peer support in treatment programs addressing substance abuse and/or mental health problems. Notably, substantial research suggests that holistic, communitybased support services are associated with positive treatment outcomes and that peer-based community support, which typically follows a participatory and empowerment approach, is an important intervention strategy for promoting long term recovery (McLellan et al., 1998).

\section{Network Therapy}

Network Therapy is a practice used in substance abuse treatment programs (Galanter, 1993) that combines psychodynamic approaches and behavioral therapy, while engaging the client in a support network composed of family members and peers. The network is managed by the therapist to provide cohesiveness and support, address treatment engagement barriers, and promote compliance with treatment. Social cohesiveness is defined as the sum of all forces that act on members of a group to keep them connected and it is argued to be an important factor in connecting a client to the therapy context, even when he or she is inclined to drop out (Zander, 1962). Galanter and Brook (2001) demonstrated the success of these programs for adults with substance abuse disorders, where both healthy peers and those who were recovering engaged 
with the client to strengthen rehabilitation. They found that network therapy was superior to individual therapy in terms of sustaining abstinence. Moreover, it appears that emotional engagement and intense relatedness with peers, which can occur in group therapy, leads to an improvement in emotional wellbeing. Similarly, Speck and Attneave (1973) used a large support group drawn from the client's family and social network as a tool for psychiatric management. Once mobilized, the network was successfully used as both psychological and practical aids in averting re-hospitalization. These studies, while they focused on adult clients, suggest opportunities for future treatment research for youth clients.

\section{Wraparound Models}

A program with a strong peer support component for youth is Wraparound Milwaukee (Kamradt, 2000). The Wraparound philosophy advocates care in which the family and the surrounding community are involved in the treatment of adolescents with emotional, mental health and behavioural needs. The Wraparound Milwaukee 'strength-based approach' focuses on both the child's individual strengths and on the strengths of the community. While the approach concerns itself more intensely with the family unit than the child's peers, both are incorporated into the model if the child requests involvement from the community or their peers. The program is flexible and allows youth to identify role models and peers that may positively influence them. Clinical outcomes have been positive, although the particular role of peer support in these outcomes has not been evaluated.

\section{Other Approaches}

A more systematic approach to understanding the value of peer support is offered by White (2004), who investigated the effectiveness of a community-oriented program with citizenship training and peer support, in conjunction with standard clinical treatment and justice 
Peer Support and Youth Recovery

diversion services. Results showed this peer-enhanced approach to treatment was superior to the standard clinical treatment and justice diversion services alone in reducing alcohol use and drug use and criminal justice charges for persons with severe mental illnesses. The peer support components were led by staff who had personal knowledge of coping with psychiatric illnesses and stigma in society. They were therefore perceived to have a unique ability to engage clients and support them in their own recovery. Participants demonstrated benefits from standard treatment as well as the citizenship and peer components. This suggests that the engagement of youth with their community can support their connection with community as well as broaden their support network. Of course, many different treatment methods were included in this study and further research is needed to clarify the specific role of peer support in producing positive outcomes.

\section{Peer-led and Peer-implemented Interventions}

In the United States, treatment for substance abuse are informed by peer-support frameworks, such as Alcoholics Anonymous (Marlatt \& Donovan, 2005). According to Marlatt and Donovan's research, peer support provides individuals with the appropriate skills and support to prevent and overcome relapse. Larimner and colleagues (2001) suggest that these types of groups encourage motivation to abstain from drinking, as studied in the context of atrisk fraternity drinkers, by providing feedback and encouragement to other members. In order to understand how peer-led support can be effective, Galanter et al.(1990) asked a group of clients, who had received both peer-led support and medically-directed care to provide information about degree of cohesiveness clients felt towards other AA members, their feelings towards the treating doctors, and towards their family members, as well as aspects of their substance use. The results indicated that respondents perceived AA as the most potent element in their recovery in 
comparison to physician counseling, family therapy, and a 'desire to do well'. The results also revealed high intensity affiliative feelings for fellow AA members. Upon further data analysis, the researchers concluded that participants found a sense of shared belief to be most influential in their recovery. Subsequent reviews, for example Emrick (1987), have found that AA is most beneficial when it is built into an extended, structured aftercare program, suggesting that both clinical/psychiatric care and peer support are important elements in recovery.

Notably, research conducted by Hunkeler et al. (2000) has directly compared services delivered by people with lived experience to those delivered by health care professionals. More specifically, 302 patients starting anti-depressant drug therapy were referred to one of two conditions - emotional support and focused behavioral interventions offered by primary care nurses or by trained peer support workers who had recovered from depression. The efficacy of these two augmentations did not differ significantly, and suggests that trained health professionals and peer support workers can be just as effective at disseminating useful information that might aid recovery. Interestingly, these researchers also found no significant effect on treatment outcomes when matching client to peer support worker by age, gender or race. It appears that trained support from individuals who genuinely appear to care, measured by patient satisfaction at the end of the treatment period, and who provide opportunities to discuss management of medication and symptoms, can be significantly supportive for recovery (Hunkeler et al., 2000).

While this study does not demonstrate that peers are superior to other treatment providers in providing effective support, these is some evidence that the inclusion of peers in existing treatment approaches may augment positive short term effects. For example, Solomon et al. (2000) were interested in the potential effects of telephone peer support on smoking cessation for 
low-income women receiving free nicotine patches and proactive telephone peer support. Telephone support from individuals who had been successful in quitting was considered an appropriate behavioral support for these women due to the limited resources that were available to them, and to the smoking culture in which they were embedded. The peer support leader initiated and followed up on calls, scheduled at convenient times for participants to ensure engagement with the client and maintain involvement in a treatment program. During the calls, the peer support leaders provided encouragement, guidance, reinforcement for quitting, and helped the women to cope with high-risk smoking situations using semi-structured protocols. The results showed that at the 10-day and three-month follow up, significantly more women in the patch and proactive telephone support condition were abstinent than in the patch-only condition. The convenience of the program, and the relatable nature of peer support workers, appeared to act as important facilitators of recovery (Solomon et al., 2000). No differences were found, however, at the 6-month follow up suggesting that the addition of peer support enhanced short-term, but not long-term, cessation.

As for sustained abstinence, peer support in the form of 'permanent supportive housing' (PSH) appears to show positive effects on recovery in the longer-term (Boisvert, Martin, Grosek and Claire, 2008). PSH involves individuals who were homeless and engaged in a peer support community (PSC) that included occupational therapy services that focused on the issues of daily living in group settings, individual and group therapy led by a trained clinician, mental health services, and medications management. The PSC program was designed using the guiding principles of occupational therapy, but was arranged by the PSH community and clients had opportunities to assume leadership roles within the community. Members of the community found that they shared similar values with others, such as: openness, helpfulness, trust and 
sobriety. They also identified common goals, such as to lead by example, and to teach others. Once the PSH community had established their own management, the community members formulated roles within the housing community to facilitate the overall wellbeing of those in the program. Bi-weekly meetings and social events promoting participation, mutual support and selfdetermination for the residents provided participants with opportunities to take control in their

lives and provided them with social supports upon discharge. Most importantly, the program had significantly reduced rate of relapse at a 9-month follow up, suggesting that with some clinical guidance, peer support may be a useful means of inspiring leadership, self-determination, perception of community affiliation, and sobriety (Boisvert et al., 2008).

From the described studies, it appears that peer support has the potential to support achievement of sustained abstinence and wellbeing for clients. Emotional support from others who have successfully engaged in the recovery process appears to increase affiliation and improve outcomes by creating common goals and shared values. Importantly, peer support can be delivered face-to-face or over the telephone, which makes the application of this type of therapy extremely broad and helpful for those who feel they may face stigma if they are associated with a community support group. There is also evidence demonstrating that peer support is a viable addition to hospital interventions, with the added benefit of long-term support once an individual leaves inpatient care. Furthermore, the effectiveness of peer support helps to shorten hospital stays and costs, which serves as a positive reciprocal relationship for all those involved.

\section{Limitations and Future Directions}

Firstly, a major limitation of the peer support literature has been methods of data collection and analysis. Often reports give detailed notes on the provision of care by mental 
health professionals but a similar level of information is rarely outlined for peer support approaches. Across studies "peer support" is defined and measured in very different ways, making it difficult to compare and/or generalize across studies. Moreover, it is difficult to reliably collect information on peer support when that support is not fully systemized nor understood (Sells et al., 2006). In addition, given the nature of peer support, specific peer support interactions are likely to be unique to the client and peer support staff involved, so creating and consistently implementing a specific a protocol is a challenge that will require further development. Furthermore, domains other than symptoms or substance use abstinence should be systematically measured, such as quality of life, and community functioning. Peer support research also needs to focus on the nature of the relationship between the peer support leaders and the clients, developing models of peer support, manuals, training curricula and fidelity measures to determine what peer providers do with their own life experiences and whom their experiences are best able to help.

Secondly, peer support workers act as a hybrid between services provided by trained professionals and naturally occurring community support (Davidson et al., 2006). However it is important that the roles of peer staff are clearly defined and articulated in the treatment setting to avoid confusion and concern amongst both clinical staff and group members. Specifically, peer support staff are hired to utilize their personal history of serious mental illness or substance abuse to enhance their credibility, to model adaptive problem solving, instill hope, and demonstrate the benefits of participation in treatment and rehabilitation. Another concern that can arise in peer support programs is potential confusion around the boundaries of friendship and a staff-client relationship. By design, peer-leaders are expected to disclose personal and intimate information about their experiences and, as such, group members may desire a level of friendship 
that is beyond the scope of the treatment setting. Davidson et al. (2006) pose the question, "How can they succeed at being friendly with clients without actually becoming friends with them?" Importantly, in this situation, confidentiality can be more challenging to maintain. The group members expect privacy from the peer support leader, whereas the expectation of the peer leader abides by the same standards of confidentiality as the rehabilitation staff (such as reporting a risk to self or others). Kennedy and Humphreys (1994) also note that peer staff and clients tend to gravitate towards more casual settings (e.g., coffee shops), which may promote friendship between the peer leader and client. In response to these concerns, Kennedy and Humphreys (1994) suggest confining meetings to hospitals or designated meeting points. Again, creating training curricula and further developing models of peer support would likely serve to decrease the confusion around issues of boundaries and friendships (Davidson et al, 2006).

Despite current gaps in peer support processes and standardization, peer support appears to have the potential to benefit clients. It is solidly grounded in psychosocial theory and has emerging evidence across settings, modalities and client populations. There is much to be gained from the hope, healthy coping, and alternative worldviews that are demonstrated by the very presence of peers with lived experience in the mental health workforce. With future research the benefits are likely only to be more fully realized. 
Peer Support and Youth Recovery

\section{References}

Berndt, T. J. (1979). Developmental changes in conformity to peers and parents. Developmental Psychology, 15(6), 608.

Birckmayer, J. D., Holder, H. D., Yacoubian, JR, G. S., \& Friend, K. B. (2004). A general causal model to guide alcohol, tobacco, and illicit drug prevention: assessing the research evidence. Journal of Drug Education, 34(2), 121-153.

Boak, A., Hamilton, H. A., Adlaf, E. M., \& Mann, R. E. Drug use among Ontario Students, 1977-2013: Detailed OSDUHS findings. CAMH Research Document Series, (36).

Boisvert, R. A., Martin, L. M., Grosek, M., \& Clarie, A. J. (2008). Effectiveness of a peer-support community in addiction recovery: participation as intervention. Occupational Therapy International, 15(4), 205-220.

Carling, P. J. (1995). Return to community: Building support systems for people with psychiatric disabilities. Guilford Press.

Chesler, M. A. (1990). The "dangers" of self-help groups: Understanding and challenging professionals' views. Working with self-help, 301-324.

Collins, R. L. (1996). For better or worse: The impact of upward social comparison on selfevaluations. Psychological Bulletin, 119(1), 51.

Davidson, L., Chinman, M., Kloos, B., Weingarten, R., Stayner, D., \& Tebes, J. K. (1999). Peer support among individuals with severe mental illness: A review of the evidence. Clinical Psychology: Science and Practice, 6(2), 165-187.

Davidson, L., Chinman, M., Sells, D., \& Rowe, M. (2006). Peer support among adults with serious mental illness: a report from the field. Schizophrenia Bulletin, 32(3), 443-450.

Deegan, P. E. (1993). Recovering our sense of value after being labeled mentally ill. Journal of Psychosocial Nursing and Mental Health Services, 31(4), 7-11.

Dennis, C. L. (2003). Peer support within a health care context: a concept analysis. International Journal of Nursing Studies, 40(3), 321-332.

Eggert, L. L., Thompson, E. A., Herting, J. R., Nicholas, L. J., \& Dicker, B. G. (1994). Preventing adolescent drug abuse and high school dropout through an intensive schoolbased social network development program. American Journal of Health Promotion, $8(3), 202-215$.

Elkind, D. (2007). The hurried child: Growing up too fast too soon. Da Capo Press.

Emrick, C. D. (1987). Alcoholics Anonymous: affiliation processes and effectiveness as treatment. Alcoholism: Clinical and Experimental Research, 11(5), 416-423. 
Festinger, L. (1954). A theory of social comparison processes. Human Relations, 7(2), 117-140.

Galanter, M. (1993). Network therapy for addiction: a model for office practice. American Journal of Psychiatry, 150, 28-28.

Galanter, M., \& Brook, D. (2001). Network therapy for addiction: bringing family and peer support into office practice. International Journal of Group Psychotherapy, 51(1: Special issue), 101-122.

Glass, T. A., De Leon, C. F. M., Bassuk, S. S., \& Berkman, L. F. (2006). Social engagement and depressive symptoms in late life longitudinal findings. Journal of Aging and Health, $18(4), 604-628$.

Hunkeler, E. M., Meresman, J. F., Hargreaves, W. A., Fireman, B., Berman, W. H., Kirsch, A. J., $\&$ Salzer, M. (2000). Efficacy of nurse telehealth care and peer support in augmenting treatment of depression in primary care. Archives of Family Medicine, 9(8), 700.

Jacobson, N., \& Curtis, L. (2000). Recovery as policy in mental health services: Strategies emerging from the states. Psychiatric Rehabilitation Journal, 23(4), 333-341.

Kamradt, B. (2000). Wraparound Milwaukee: Aiding Youth with Mental Health Needs. Juvenile Justice, 7(1).

Kennedy, M., \& Humphreys, K. (1994). Chapter 8. Understanding worldview transformation in members of mutual help groups. Journal of Prevention \& Intervention in the Community, 11(1), 181-198.

Larimer, M. E., Turner, A. P., Anderson, B. K., Fader, J. S., Kilmer, J. R., Palmer, R. S., \& Cronce, J. M. (2001). Evaluating a brief alcohol intervention with fraternities. Journal of Studies on Alcohol and Drugs, 62(3), 370.

Marlatt, G. A., \& Donovan, D. M. (Eds.). (2005). Relapse prevention: Maintenance strategies in the treatment of addictive behaviors. Guilford Press.

McLellan, A. T., Hagan, T. A., Levine, M., Gould, F., Meyers, K., Bencivengo, M., \& Durell, J. (1998). Supplemental social services improve outcomes in public addiction treatment. Addiction, 93(10), 1489-1499.

Miller, P. H. (2010). Theories of developmental psychology. Macmillan.

O'Connell, M. E., Boat, T., \& Warner, K. E. (Eds.). (2009). Preventing Mental, Emotional, and Behavioral Disorders Among Young People: Progress and Possibilities. National Academies Press.

Roman, P. M., \& Trice, H. M. (1970). The development of deviant drinking behavior. Archives of Environmental Health: An International Journal, 20(3), 424-435. 
Rosenstock, I. M., Strecher, V. J., \& Becker, M. H. (1988). Social learning theory and the health belief model. Health Education \& Behavior, 15(2), 175-183.

Sells, D., Davidson, L., Jewell, C., Falzer, P., \& Rowe, M. (2006). The treatment relationship in peer-based and regular case management for clients with severe mental illness. Psychiatric Services, 57(8), 1179-1184.

Shillington, A. M., Lehman, S., Clapp, J., Hovell, M. F., Sipan, C., \& Blumberg, E. J. (2005). Parental monitoring: Can it continue to be protective among high-risk adolescents? Journal of Child \& Adolescent Substance Abuse, 15(1), 1-15.

Solomon, L. J., Secker-Walker, R. H., Flynn, B. S., Skelly, J. M., \& Capeless, E. L. (2000). Proactive telephone peer support to help pregnant women stop smoking. Tobacco Control, 9(suppl 3), iii72-iii74.

Speck, R. V., \& Attneave, C. L. (1973). Family networks. Pantheon.

Taylor, S. E., \& Lobel, M. (1989). Social comparison activity under threat: downward evaluation and upward contacts. Psychological Review, 96(4), 569.

Tobler, N. S. (1992). Drug prevention programs can work: Research findings. Journal of Addictive Diseases, 11(3), 1-28.

Vaughan, C. A., Foshee, V. A., \& Ennett, S. T. (2010). Protective effects of maternal and peer support on depressive symptoms during adolescence. Journal of Abnormal Child Psychology, 38(2), 261-272.

Ward, J., Hunter, G., \& Power, R. (1997). Peer education as a means of drug prevention and education among young people: an evaluation. Health Education Journal, 56(3), 251263.

White, W. (2004). The history and future of peer-based addiction recovery support services. Prepared for the SAMHSA Consumer and Family Direction Initiative, 22-23.

White, W. L., Kurtz, E., \& Sanders, M. (2006). Recovery management. Great Lakes Addiction Technology Center (ATTC) Network, Jane Addams College of Social Work.

Wills, T. A. (1981). Downward comparison principles in social psychology. Psychological Bulletin, 90(2), 245.

Wood, J. V. (1989). Theory and research concerning social comparisons of personal attributes. Psychological Bulletin, 106(2), 231.

Zander, A. F. (1962). Group dynamics: Research and theory. Row, Peterson.

Zucker, R. A. (1979). Developmental aspects of drinking through the young adult years. In Youth, alcohol, and social policy (pp. 91-146). Springer US. 